\title{
Variation in receipt of therapy and survival with provider volume for medical oncology in non-curative esophago-gastric cancer: a population-based analysis
}

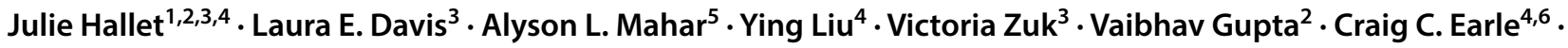 \\ Natalie G. Coburn ${ }^{1,2,3,4}$
}

Received: 20 May 2019 / Accepted: 3 October 2019 / Published online: 18 October 2019

(c) The International Gastric Cancer Association and The Japanese Gastric Cancer Association 2019

\begin{abstract}
Background While surgical care by high-volume providers for esophago-gastric cancer (EGC) yields better outcomes, volume-outcome relationships are unknown for systemic therapy. We examined receipt of therapy and outcomes in the non-curative management of EGC based on medical oncology provider volume.

Methods We conducted a population based retrospective cohort study of non-curative EGC over 2005-2017 by linking administrative healthcare datasets. The volume of new EGC consultations per medical oncology provider per year was calculated and divided into quintiles. High-volume (HV) medical oncologists were defined as the 4-5th quintiles. Outcomes were receipt of chemotherapy and overall survival (OS). Multivariate logistic and Cox-proportional hazards regressions examined the association between management by HV medical oncologist, receipt of systemic therapy, and OS.

Results 7011 EGC patients with non-curative management consulted with medical oncology. 1-year OS was superior for HV medical oncologists (> 11 patients/year), with $28.4 \%$ (95\% CI 26.7-30.2\%) compared to $25.1 \%$ (95\% CI 23.8-26.3\%) for low volume $(p<0.001)$. After adjusting for age, sex, comorbidity burden, rurality, income quintile, and diagnosis year, HV medical oncologist was independently associated with higher odds of receiving chemotherapy (OR 1.13, 95\% CI 1.01-1.26), and independently associated with superior OS (HR 0.89, 95\% CI 0.84-0.93).

Conclusions Medical oncology provider volume was associated with variation in non-curative management and outcomes of EGC. Care by an HV medical oncologist was independently associated with higher odds of receiving chemotherapy and superior OS, after adjusting for case mix. This information is important to inform disease care pathways and care organization; an increase in the number of HV medical oncologists may reduce variation and improve outcomes.
\end{abstract}

Keywords Esophagus $\cdot$ Stomach $\cdot$ Cancer $\cdot$ Outcomes $\cdot$ Volume

Part of this work was presented at the Annual Meeting of the Canadian Society of Surgical Oncology held in Toronto, ON, on May 3rd 2019, and submitted for presentation at the Canadian Surgery Forum to be held in Montréal, QC, in September 2019.

Electronic supplementary material The online version of this article (https://doi.org/10.1007/s10120-019-01012-z) contains supplementary material, which is available to authorized users.

Julie Hallet

Julie.hallet@sunnybrook.ca

1 Division of Surgical Oncology, Odette Cancer CentreSunnybrook Health Sciences Centre, 2075 Bayview avenue, T2-063, Toronto, ON M4N 3M5, Canada

2 Department of Surgery, University of Toronto, Toronto, ON, Canada

\section{Introduction}

The new face of cancer care includes an increasing complexity of cases and therapeutic options. As therapies improve, decision-making and care delivery get more challenging, such that clinical experience is becoming ever more crucial.

\footnotetext{
Sunnybrook Research Institute, Toronto, ON, Canada

ICES, Toronto, ON, Canada

5 Department of Community Health Sciences, University of Manitoba, Winnipeg, MB, Canada

6 Division of Medical Oncology, Odette Cancer Centre-Sunnybrook Health Sciences Centre, Toronto, ON, Canada
} 
There is a large body of literature supporting the importance of clinical volume to obtain better short and long-term outcomes for cancer surgery $[1,2]$. These observations have led to institution and system-level policy changes to concentrate specialized cancer surgery services to high-volume settings with subsequent gains in outcomes [3-7].

Other aspects of cancer care, such as systemic therapy, may also benefit from high volume provision of care, but are rarely examined from the volume-outcome perspective. The few studies addressing the impact of clinical volume on quality of care and survival are mostly limited to hematologic cancers, including multiple myeloma, chronic lymphoid leukemia, and lymphoma [8-12]. In 2001, the Institute of Medicine recommended that volume-outcome relationships be examined for a wide range of cancers and for all therapies, medical and surgical [13]. Over 15 years later, the impact of clinical volume on care patterns and survival remains unascertained for systemic therapy in solid cancers.

Esophago-gastric cancers (EGC) are amongst the most lethal and debilitating malignancies worldwide [14]. The majority of patients with EGC present at advanced stages when curative therapy with resection or definitive chemoradiation is no longer possible. The cornerstone of non-curative management of EGC is systemic therapy to improve symptom profile, delay progression, and prolong survival [15-20]. As therapies got more effective and yielded better outcomes, they also became more toxic, thereby increasing the complexity of care with regard to both the decision to initiate treatment and the delivery of therapy that may be impacted by clinical experience. Furthermore, EGC potentially suffers from nihilism related to its traditionally guarded prognosis, which may influence the assessment of the risk-benefit profile of systemic therapy for medical oncologists with less experience. For these reasons, the noncurative management of EGC may particularly benefit from clinical care by higher volume medical oncologists.

We conducted a population-based study to assess the association between medical oncology provider volume and receipt of therapy and survival in the non-curative management of EGC. We hypothesized that there is variability in provision of care and outcomes, and that patients cared for by high-volume medical oncologists have better survival.

\section{Methods}

\section{Study design}

Using data linked from prospectively maintained administrative databases stored at ICES in Ontario, Canada, we conducted a population-based cohort study. Under the Canada Health Act, the Ontario population benefits from universally accessible and publicly funded health care though Ontario
Health Insurance Plan (OHIP) [21]. All residents of Ontario are eligible for OHIP after they have resided in the province for 3 months.

The study was approved by the Sunnybrook Health Sciences Centre Research Ethics Board. It was conducted and reported following the REporting of studies Conducted using Observational Routinely collected Data (RECORD) statement [22].

\section{Data sources}

This study linked administrative data sets at ICES using unique Identification Key Number for each patient (IKN). The Ontario Cancer Registry (OCR) includes all patients with a cancer diagnosis (excluding non-melanoma skin cancer) in Ontario since 1964 [23]. The reliability of its data has previously been reported [21-23]. The Registered Persons Database (RPDB) contains vital status and demographic data on all individuals covered under OHIP [24]. Information regarding health services is included in the Canadian Institute of Health Information Discharge Abstract Database (CIHI-DAD) for acute inpatient hospitalizations, the National Ambulatory Care Reporting System for same-day surgery admissions, emergency room visits and oncology clinic visits, and the OHIP Claims Database for billing from health care providers, including physicians, groups, laboratories, and out-of-province providers [25]. The Cancer Activity Level Reporting (ALR) database is maintained by the OCR and includes chemotherapeutics and medications administered to cancer patients. These databases have been validated for a variety of diagnoses and services [25].

The data sets were linked using unique encoded identifiers and analyzed at ICES. The research team's analyst (YL) had complete access to all the data sets used in this study to create the study cohorts, proceed to linkage, and perform the analyses.

\section{Study population and cohort}

This study was conducted in all patients with a valid OHIP number diagnosed with EGC from January 1st, 2005 to March 31st, 2017. Patients with a new diagnosis of esophageal or gastric adenocarcinoma or squamous cell carcinoma were identified in the OCR using International Classification of Diseases for Oncology (ICD-O.3) codes, and we retained only patients who did not undergo a curative-intent esophagectomy or gastrectomy or did not receive curativeintent chemoradiation therapy from 180 days prior to the date of diagnosis to the end of follow-up (Supplementary Table 1). Patients were excluded if they died before or on the date of diagnosis, had another cancer diagnosis in the 5 years prior or at any time following EGC diagnosis, or were aged $<18$ or $>99$ years at the time of diagnosis. Of 
those, patients were included in this provider-volume analysis if they were seen by a medical oncologist between the date of diagnosis and the end of follow-up (date of death, last clinical encounter, or end of study as of March 31st, 2018), defined using OHIP claims. A physician submitting billings for chemotherapy administration codes (G381, G281, G339, G345, G359) at any time during the study period was classified as a medical oncologist [25].

\section{Exposure}

The main exposure of interest was the provider volume of the medical oncologist seeing each patient in the cohort. Provider volume was computed as the number of new noncurative EGC consultations seen per medical oncologist per year over the study period. Each patient was assigned to one medical oncologist; if a patient saw more than one medical oncologist, the medical oncologist seen most often was assigned. Patients were divided into quintiles based on the provider volume of their medical oncologist to evaluate variability in outcomes across a continuum of provider volume. High volume was defined as the 4 th and 5 th quintiles (highest). Quintiles were chosen as a measure of high volume as suggested in the previous studies on surgical clinical volume $[1,2]$.

\section{Outcomes measures}

Receipt of chemotherapy was defined by identifying patients for whom at least two chemotherapy infusions were billed from the date of diagnosis to end of follow-up. The identification of chemotherapy administration using OHIP has previously been described and used, with $90 \%$ concordance between OHIP codes and patient medical records (Cancer Activity Level Reporting) [26-28]. Overall survival (OS) was measured from date of diagnosis to the date of death according to the RPDB. The end of follow-up was defined as the date of death, the date of last contact, or March 31, 2017, offering an opportunity for a minimum of 12 month follow-up for all patients. Using population-based administrative healthcare databases, there was no loss to follow up in this cohort.

\section{Covariates}

Age and sex were obtained from the RPDB. Rural living was determined with postal code of residence [29]. Income quintile was assessed with an ecologic measure based on the median income of a patient's postal code of residence using national census data $[30,31]$. The comorbidity burden was measured using the Elixhauser comorbidity index [32]. The index includes 31 comorbidities identified using ICD-9 and 10 codes with a 2-year look-back window and was dichotomized with a cutoff of 4 for high comorbidity burden, as previously suggested [33].

\section{Statistical analysis}

Categorical variables were described as absolute number $(n)$ and proportion (\%), and continuous variables as means with standard deviation (SD) or median with inter-quartile range (IQR). Comparison testing between high and low-volume medical oncologists groups was undertaken with Chi-square test for categorical variables, and the Kruskal-Wallis or $t$ test for continuous variables. Kaplan-Meier methods with comparison using the log-rank test were used for OS analysis [34].

The median proportion of patients receiving chemotherapy, median number of chemotherapy cycles per patient, and median OS were evaluated across provider-volume quintiles to appreciate variability. To test significance of variation based on provider volume, the equality of variance was assessed across quintile group using the Brown-Forsythe-Levene's test to determine if more variation exists than would be expected by chance alone [35].

Multivariable regression models were constructed to assess the association between provider volume and outcomes. Relevant demographic and clinical characteristics were identified a priori as potential confounders of the relationship between provider volume and outcomes. The variables were selected based on clinical relevance (markers of complexity of cancer care) and existing literature (known relationship with OS for EGC) [36, 37]. The most parsimonious set of covariates was selected. The following covariates were included: age (categorical), sex, comorbidity burden, income, rural living, time period of diagnosis (2005-2010 vs. 2011-2017), and type of cancer (esophageal vs. gastric). Logistic regression was used for receipt of chemotherapy and Cox proportional hazards regression for OS, and results reported as odds ratio (OR) and hazard ratios (HR) with $95 \%$ confidence intervals $(95 \% \mathrm{CI})$, respectively.

Considering the high-fatality of EGC, higher-risk patients may die before having the opportunity to see a high-volume medical oncologist or may not be well enough to seek care by a high-volume medical oncologist. We conducted a sensitivity analysis by restricting the cohort to patients surviving a minimum of 30 days following the date of diagnosis.

Statistical significance was set at $p \leq 0.05$. All analyses were conducted using SAS Enterprise Guide 6.1 (SAS Institute, Cary, NC, USA).

\section{Results}

A total of 7011 patients were identified with a new diagnosis of EGC, who did not undergo surgery, and had a medical oncology consultation (Supplementary Fig. 1). Median 
follow-up was 6.3 (IQR 1.9-11.9) months. Overall, 30.7\% $(n=2154)$ of patients received chemotherapy. The most common first-line regimens administered over the study period were cisplatinum monotherapy in $45.5 \%(n=980)$, 5-FU monotherapy in $28.7 \%(n=619)$, and cisplatinum combination therapy in $19.1 \%(n=411)$. Patients receiving chemotherapy had longer median survival than those who did not receive chemotherapy, with 9.4 (IQR 5.4-15.4) months compared to 3.8 (IQR 1.9-8.4) months $(p<0.001)$ (Supplementary Fig. 2).

The patient volume of the 361 identified medical oncologists ranged from 1 to 23 per year (Supplementary Table 2). The 4 th provider-volume quintile cutoff was 11 patients/ year; therefore, high volume was defined as greater than or equal to 11 patients per year (Supplementary Table 3). With this definition, $36(10.0 \%)$ medical oncologists were high volume (Fig. 1), and 2518 (35.9\%) patients were cared for by high-volume medical oncologists. While numbers cannot be reported due to confidentiality and privacy guidelines for managing small cells $(n<6)$, no difference was observed in medical oncologists' demographic, training, or practice characteristics, between high- and low-volume medical oncologists.

Characteristics of included patients are presented in Table 1. Patients cared for by high-volume medical oncologists were more likely to be of younger age, of male sex, and be diagnosed in more recent years. No difference was observed between groups regarding rural residence and income quintile.

The median proportion of patients receiving chemotherapy varied across quintiles of provider volume, ranging from
$47.1 \%$ in the 1 st quintile to $51.4 \%$ in the 4 th quintile (high volume). The narrower IQR with increasing provider-volume quintiles indicated reduction in variation with increasing provider volume (Fig. 2). The Brown-Forsythe-Levene test showed significant differences in variance across quintiles $(p<0.01)$. Patients treated by medical oncologists in the higher volume quintile received more chemotherapy cycles, with median 5 (IQR 1-13) for 1st quintile, 6 (IQR 2-15) for 2nd quintile, 6 (IQR 2-15) for 3rd quintile, 8 (IQR 3-17.5) for 4th quintile, and 7 (IQR 3-18) for 5th quintile (Brown-Forsythe-Levene $p<0.001$ ). After adjusting for age, sex, comorbidity burden, income, rural residence, and time period of diagnosis, care by a high-volume medical oncologist, there was associated with higher odds of receiving chemotherapy (OR 1.13, 95\% CI 1.01-1.26).

Median survival also varied across quintiles of provider volume, with superior survival in the 4th and 5th quintiles (7.1 and 7.0 months, respectively) compared to the $1 \mathrm{st}-3 \mathrm{rd}$ quintiles (6.2-6.6 months) (Fig. 3). Evaluation of IQRs shows reduced ranges in higher quintiles. However, the Brown-Forsythe-Levene test did not reach statistical significance $(p=0.16)$. OS was superior for patients seeing highvolume medical oncologists, with 1-year OS of $28.4 \%$ (95\% CI $26.7-30.2 \%$ ) compared to $25.1 \%$ (95\% CI $23.8-26.3 \%$ ) for low volume (Fig. 4) $(p<0.001)$. After adjusting for age, sex, comorbidity burden, income, rural residence, and time period of diagnosis, receipt of care by a high-volume medical oncologist was independently associated with superior OS (HR 0.89, 95\% CI 0.84-0.93).

When restricting the analysis to patients who survived a minimum of 30 days following diagnosis $(n=6605)$,
Fig. 1 Distribution of medical oncologists by provider volume (patients/year) $(n=361$ medical oncologists)

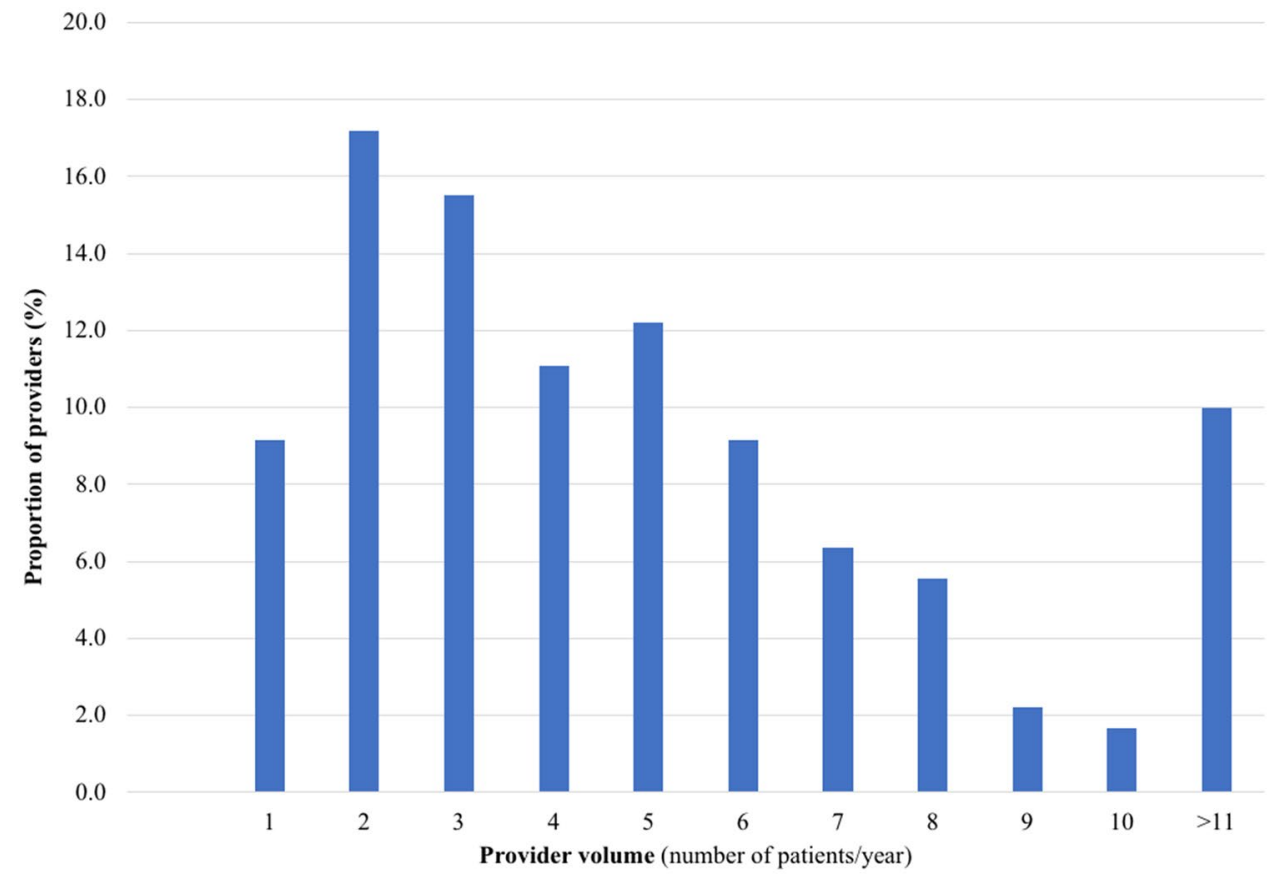


Table 1 Characteristics of patients with non-curative management of esophagogastric cancer, stratified by receipt of care by low- and highvolume medical oncologists

\begin{tabular}{lclc}
\hline Variable & $\begin{array}{l}\text { Low-volume medical oncolo- } \\
\text { gists }(<11 \text { patients/year) } \\
n=4493\end{array}$ & $\begin{array}{l}\text { High-volume medical oncolo- } \\
\text { gists }(>11 \text { patients/year) } \\
n=2518\end{array}$ & $p$ value \\
\hline Cancer type & & & \\
Esophageal & $1423(31.7 \%)$ & $997(39.6 \%)$ & $<0.001$ \\
Gastric & $3070(68.3 \%)$ & $1521(60.4 \%)$ & $<0.001$ \\
Age (years old) & $1187(26.4 \%)$ & $794(31.5 \%)$ & $<0.001$ \\
$<60$ & $1156(25.7 \%)$ & $699(27.8 \%)$ & 0.23 \\
61-70 & $1213(27.0 \%)$ & $643(25.5 \%)$ & 0.89 \\
$71-80$ & $937(20.9 \%)$ & $382(15.2 \%)$ & \\
$>81$ & $1496(33.3 \%)$ & $246(9.8 \%)$ & 0.16 \\
Female sex & $480(10.7 \%)$ & $326(12.9 \%)$ & \\
High comorbidity burden & & & \\
(Elixhauser index $>4)$ & $592(13.2 \%)$ & $509(20.2 \%)$ & \\
Rural residence & & $521(20.7 \%)$ & \\
Income quintile & $946(21.1 \%)$ & $526(20.9 \%)$ & \\
1st (lowest) & $993(22.1 \%)$ & $499(19.8 \%)$ & \\
2nd & $908(20.2 \%)$ & $463(18.4 \%)$ & \\
3rd & $869(19.3 \%)$ & $1405(55.8 \%)$ & \\
4th & $777(17.3 \%)$ & & \\
5th (highest) & $2190(48.7 \%)$ & & \\
Time period of diagnosis & $2303(51.3 \%)$ & & \\
2005-2011 & & & \\
2012-2017 & & & \\
\hline
\end{tabular}

Values are $n(\%)$ representing column percentages

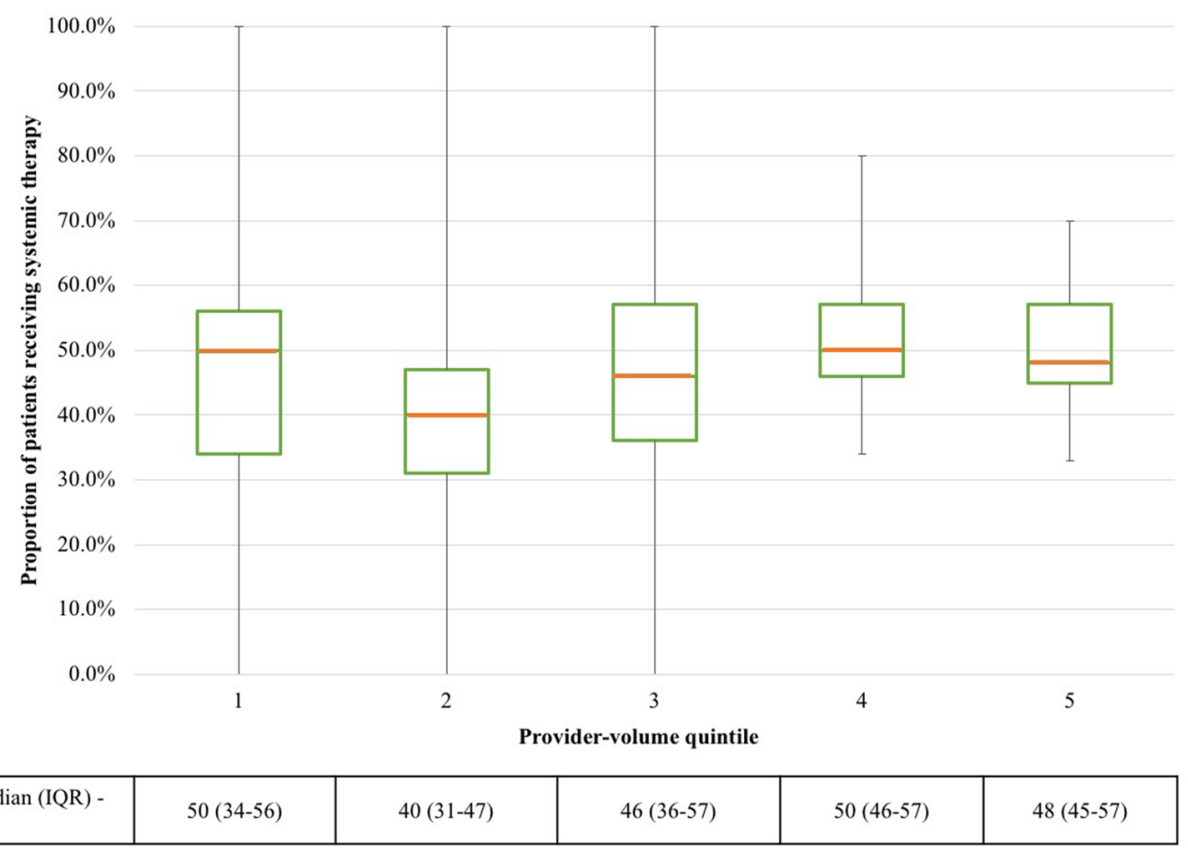


Fig. 3 Median overall survival (months) stratified by providervolume quintiles (patients/year). Median is at the orange line.

Boxes represent inter-quartile range, and vertical lines represent range

Fig. 4 Overall survival stratified by receipt of care by low- and high-volume medical oncologists (Kaplan-Meier methods)
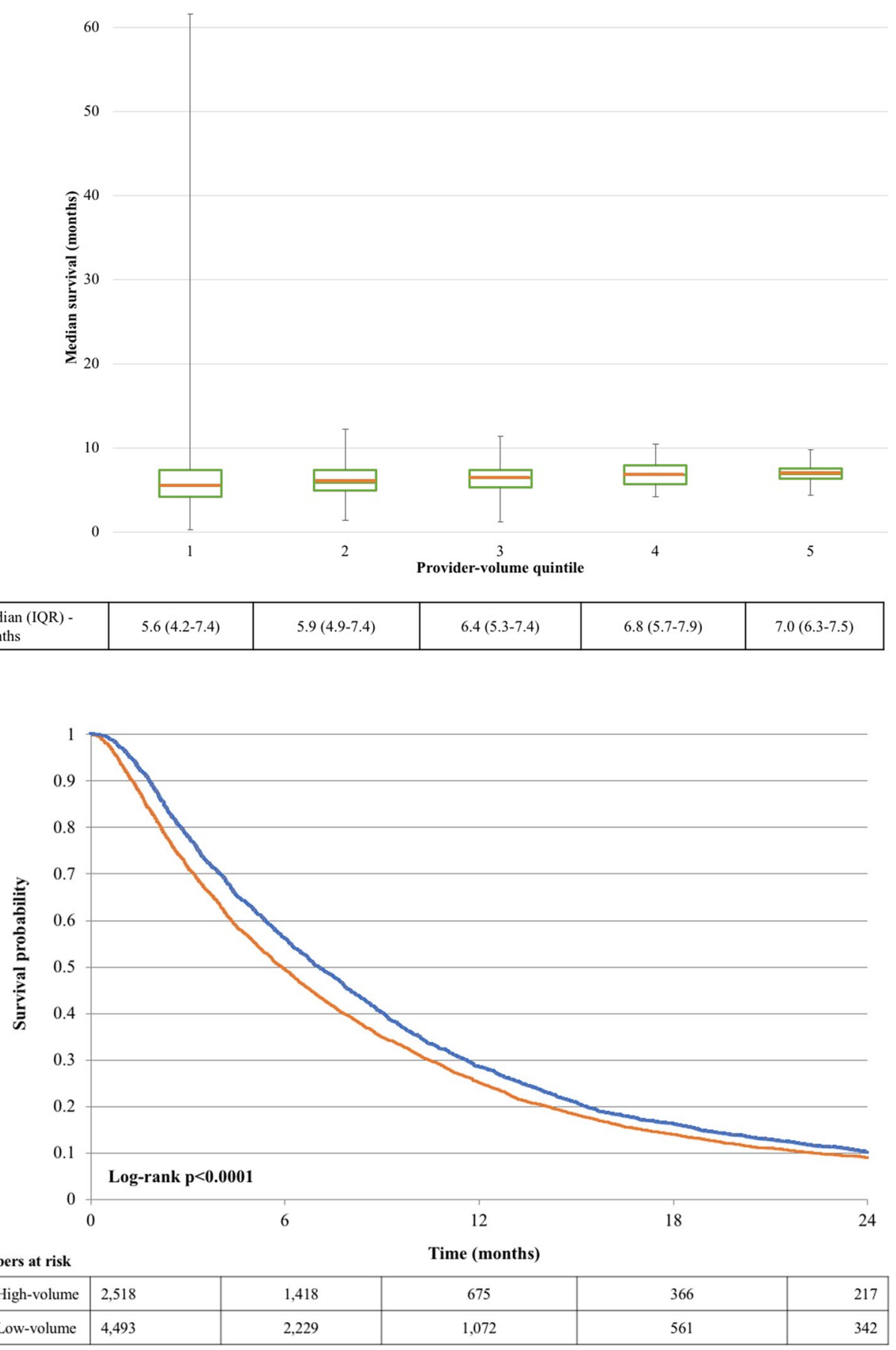

care by high-volume medical oncologists was not independently associated with receipt of chemotherapy (OR 1.06, 95\% CI 0.95-1.19). However, high-volume medical oncologists remained independently associated with superior OS (HR 0.92, 95\% CI 0.87-0.97).

\section{Discussion}

In this novel population-based analysis of systemic therapy delivery for non-curative EGC, we observed significant variation in receipt of therapy with increasing medical oncologists' provider volume. Within this variation, we identified a clinically relevant cutoff to define high-volume medical oncologists, which is associated with $13 \%$ higher odds of 
receiving systemic therapy and superior OS after, when adjusting for case-mix.

The current analysis is an important addition to the literature, as it provides the first assessment of the relationship between provider volume, therapy, and survival in a solid cancer, EGC. The magnitude of the OS benefit with highvolume medical oncologists is similar than that observed for high-volume cancer surgery [1]. Prior studies of volume outcome for systemic therapy are limited to hematologic cancers and outlined increasing OS with increasing patient volume at the institution and population levels [8, 10-12]. One study used the mean number of patients per medical oncologists as a covariate to examine the odds of early termination of adjuvant therapy for colorectal cancer, but did not assess the association with patient selection for therapy or survival [38]. Thus, volume-outcome relationships between high compared to low-volume medical oncologists had not been comprehensively addressed for solid cancers previously, likely owing to the difficulty to access good quality data on systemic therapy that is often delivered in private or semi-private outpatient centres, as highlighted by the Institute of Medicine [13]. We herein leveraged unique comprehensive and complete healthcare data for an entire population to overcome this limitation and address an important clinical question.

With a high-fatality disease such as EGC, any gain in survival can be significant for patients [14]. Systemic therapy is recommended for unresectable advanced or metastatic EGC with ECOG of 2 or better [15-17, 19, 20, 39]. Access to systemic therapy is thus critical in optimizing outcomes for non-curative EGC. While the absolute difference in receipt of therapy between low- and high-volume medical oncologists may not appear large, the significant variation in delivery of therapy across provider-volume quintile points to disparities in care. This translates to a survival difference depending on whether patients consult with a low- or a highvolume medical oncologist. The magnitude of the survival benefit seems clinically important; the HR is comparable to that observed for systemic therapies that are now standard of care $[15,20,40]$. It is acknowledged that non-receipt of therapy may be acceptable if not medically feasible or not aligned with patients' preferences. Although this analysis could not decipher the factors underlying the decision to treat or not, we adjusted for patient-level characteristics that could affect this decision. In addition, there can be biases in referrals, whereby more motivated patients may seek highvolume medical oncologists, or be referred from a lowvolume to a high-volume medical oncologist, which could not be addressed in the current study. Even after restricting the analysis to 30-day survivors, the OS benefit for care by high-volume medical oncologists persisted. Nevertheless, the observed variation and differences in outcomes indicate disparities in EGC care that should be addressed so that all patients are offered the same opportunities for care. Though provider volume is only one of multiple factors underlying variation in care and outcomes, and the surgical cancer care experience suggests that favoring high-volume care can optimize therapy delivery and outcomes [41].

The most important component of variation in healthcare has been cited as "unwarranted variation", which refers to the proportion of variation that cannot be explained by differences in the patient population, but rather by the quality, appropriateness, and efficiency of care [42]. During the study period, medical oncologists had equal access to drugs under the universal healthcare coverage. Case mix also plays a role in observed variation, but does not account for all of it. Indeed, decision-making and physician preferences are known to lead to care variation, owing mostly to misinterpretation or misapplication of evidence and clinical information [43, 44]. The clinical assessment of prognosis for a high-fatality malignancy and the perception of a regimen's risk-benefit could impact the use of systemic therapy for EGC and be affected by clinical experience and provider volume.

High volume in itself does not produce good or bad outcome,s but is rather a surrogate measure for other factors such as clinical experience, processes of care, team organization, patient support, or access to clinical trials [45, 46]. Establishing disparities in the receipt of therapy and outcomes was the first step towards improving organization of cancer care based on clinical volume for medical therapy. We focused on a provider-level volume metric to make the results actionable within institutions. Moreover, we combined esophageal and gastric adenocarcinoma and squamous carcinoma, as these diseases are often treated by similar medical oncologists, such that we could provide a pragmatic approach to provider volume from a disease-site perspective. Further work is now required to determine what is different about high-volume medical oncologists. Such understanding will help create a system that leverages the benefits of care by high-volume medical oncologists to enhance patient outcomes. Focusing on high-volume medical oncologists can be compatible with care close to home that is valued by patients $[47,48]$ : reducing the overall number of medical oncologists for EGC would increase the number of high-volume medical oncologists that can then be established across a jurisdiction. Disease specialization within institutions can also lead to increased volume while maintaining geographic access. This approach has been shown to yield better outcomes for hematologic cancers [12, 49].

There are some study limitations. Due to the retrospective and administrative natures of the work, the data used were not collected specifically to answer the research question. As a result, we lacked some information regarding disease characteristics, such as extent and volume of tumor, that can impact decision making. The definition of high volume 
relied on quintile. The 4th and 5th quintiles were combined based on reduced variation in outcomes in those quintiles and the number of providers meeting this yearly patient volume. The latter was to ensure that the results would be clinically meaningful and more easily translatable to clinical practice; if too few providers met the volume criteria, the findings would be more difficult to implement. Other strategies have been used to define high volume [45]. The exact number defined herein may not perform the same in other jurisdictions. The absolute cutoff may differ depending on the health system and practice setting, especially in jurisdictions with higher incidence and clinical volume of EGC; however, the observations regarding a high-volume practice concept remain valid. Finally, this study was conducted within the Canadian universally accessible and funded healthcare system, which can impact generalizability to other systems. However, prior work has shown that the attitudes of medical oncologists regarding the delivery of systemic therapy does not differ between private and public systems, despite differences in accessing and funding drugs [50]. Furthermore, the literature on surgical volume outcome has yielded similar conclusions in various health systems across Canada, the USA and Europe, supporting the generalizability of such findings $[3,4,6,7]$.

The true population-based design of this study is a strength that allowed an assessment of all patients with EGC with continuous data collection within a universal healthcare system, where insurance status does not confound access to and receipt of care. This analysis relies on similar methodology used to establish the benefits of high-volume surgical care using routinely collected administrative data $[1,2]$. It has shown a plausible volume-outcome relationship in provision of systemic therapy for the non-curative management of EGC, and clinically significant survival differences associated with high-volume care provision, thus meeting two criteria outlined by the Institute of Medicine for the implementation of health policies on clinical volumes [13].

\section{Conclusion}

This large population-based analysis identified significant variation in receipt of therapy and OS based on provider volume in the non-curative management of EGC. Care by a high-volume medical oncologist was independently associated with increased odds of receiving systemic therapy and superior OS, after adjusting for case mix. This novel information is important to support the design of care pathways that minimize variation and enhance efficiency of care, and improve outcomes. Future work is warranted to identify consistent volume-outcome relationships for systemic therapy, consider institutional and system-level interventions to facilitate care delivery by high-volume medical oncologists.

Acknowledgements This study was supported by the Canadian Institute of Health Research (FRN \#154131) and the Sherif and Mary-Lou Hanna Chair in Surgical Oncology Research. This study was supported by ICES, which is funded by an annual grant from the Ontario Ministry of Health and Long-Term Care (MOHLTC). The opinions, results and conclusions reported in this paper are those of the authors and are independent from the funding sources. No endorsement by ICES or the Ontario MOHLTC is intended or should be inferred. Parts of this material are based on data and information compiled and provided by CIHI. However, the analyses, conclusions, opinions, and statements expressed herein are those of the author, and not necessarily those of CIHI. Parts of this material are based on data and information provided by Cancer Care Ontario (CCO). The opinions, results, view, and conclusions reported in this paper are those of the authors and do not necessarily reflect those of CCO. No endorsement by $\mathrm{CCO}$ is intended or should be inferred.

Funding This study was supported by the Canadian Institute of Health Research (FRN \#154131).

\section{Compliance with ethical standards}

Conflict of interest Julie Hallet has received speaking honoraria from Ipsen Biopharmaceuticals Canada and Novartis Oncology. Natalie Coburn receives salary support from Cancer Care Ontario as the Clinical Lead of Patient Reported Outcomes and Symptom Management. Laura Davis declares that she has not conflict of interest. Alyson Mahar declares that she has not conflict of interest. Ying Liu declares that she has not conflict of interest. Vaihbav Gupta declares that he has not conflict of interest. Craig Earle declares that he has not conflict of interest.

Ethical approval All procedures performed in studies involving human participants were in accordance with the ethical standards of the institutional and/or national research committee and with the 1964 Helsinki declaration and its later amendments or comparable ethical standards.

Informed consent Informed consent was waived by the Sunnybrook Health Sciences Centre Research Ethics Board, for the conduct of population-based analysis.

\section{References}

1. Bilimoria KY, Bentrem DJ, Feinglass JM, Stewart AK, Winchester DP, Talamonti MS, et al. Directing surgical quality improvement initiatives: comparison of perioperative mortality and long-term survival for cancer surgery. J Clin Oncol. 2008;26:4626-33.

2. Birkmeyer JD, Stukel TA, Siewers AE, Goodney PP, Wennberg DE, Lucas FL. Surgeon volume and operative mortality in the United States. N Engl J Med. 2003;349:2117-27.

3. Birkmeyer JD, Siewers AE, Marth NJ, Goodman DC. Regionalization of high-risk surgery and implications for patient travel times. JAMA. 2003;290:2703-8.

4. Simunovic M, Urbach D, Major D, Sutradhar R, Baxter N, To T, et al. Assessing the volume-outcome hypothesis and region-level quality improvement interventions: pancreas cancer surgery in two Canadian Provinces. Ann Surg Oncol. 2010;17:2537-44. 
5. Gasper WJ, Glidden DV, Jin C, Way LW, Patti MG. Has recognition of the relationship between mortality rates and hospital volume for major cancer surgery in California made a difference?: a follow-up analysis of another decade. Ann Surg. 2009;250:472-83.

6. Dikken JL, Dassen AE, Lemmens VEP, Putter H, Krijnen P, van der Geest L, et al. Effect of hospital volume on postoperative mortality and survival after oesophageal and gastric cancer surgery in the Netherlands between 1989 and 2009. Eur J Cancer. 2012;48:1004-133.

7. Fischer C, Lingsma H, Klazinga N, Hardwick R, Cromwell D, Steyerberg E, et al. Volume-outcome revisited: the effect of hospital and surgeon volumes on multiple outcome measures in oesophago-gastric cancer surgery. PLoS ONE. 2017; 12:e0183955.

8. Ireland C, Wiedower E, Mirza M, Crawley M, Tran A, Yaghmour G, et al. Impact of provider volume on outcomes of patients with hodgkin lymphoma. World J Oncol. 2018;9:46-9.

9. Huntington SF, Hoag JR, Zhu W, Wang R, Zeidan AM, Giri S, et al. Oncologist volume and outcomes in older adults diagnosed with diffuse large B cell lymphoma. Cancer. 2018;64:786-4220.

10. Go RS, Bartley AC, Crowson CS, Shah ND, Habermann EB, Holton SJ, et al. Association between treatment facility volume and mortality of patients with multiple myeloma. J Clin Oncol. 2017;35:598-604.

11. Go RS, Al-Hamadani M, Shah ND, Crowson CS, Holton SJ, Habermann EB. Influence of the treatment facility volume on the survival of patients with non-Hodgkin lymphoma. Cancer. 2016;122:2552-9.

12. Shanafelt TD, Kay NE, Rabe KG, Inwards DJ, Zent CS, Leis $\mathrm{JF}$, et al. Hematologist/oncologist disease-specific expertise and survival: lessons from chronic lymphocytic leukemia (CLL)/small lymphocytic lymphoma (SLL). Cancer. 2012;118:1827-37.

13. Institute of Medicine (US), and National Research Council (US) National Cancer Policy Board, Hewitt M, Petitti D. Interpreting the volume-outcome relationship in the context of cancer care. Washington (DC): National Academies Press; 2001.

14. Global Burden of Disease Cancer Collaboration, Fitzmaurice C, Akinyemiju TF, Lami Al FH, Alam T, Alizadeh-Navaei R, et al. Global, regional, and national cancer incidence, mortality, years of life lost, years lived with disability, and disability-adjusted life-years for 29 cancer groups, 1990 to 2016: a systematic analysis for the Global Burden of Disease Study. JAMA Oncol. 2018;4:1553-688.

15. Smyth EC, Verheij M, Allum W, Cunningham D, Cervantes A, Arnold D, et al. Gastric cancer: ESMO Clinical Practice Guidelines for diagnosis, treatment and follow-up. Ann Oncol. 2016;27:v38-v49.

16. Muro K, Van Cutsem E, Narita Y, Pentheroudakis G, Baba E, Li J, et al. Pan-Asian adapted ESMO Clinical Practice Guidelines for the management of patients with metastatic gastric cancer; a JSMO-ESMO initiative endorsed by CSCO, KSMO, MOS SSO and TOS. Ann Oncol. 2018;30:19-33.

17. Ajani JA, D'Amico TA, Almhanna K, Bentrem DJ, Besh S, Chao $\mathrm{J}$, et al. Esophageal and esophagogastric junction cancers, version 1.2015. J Natl Compr Canc Netw. 2015;13:194-227.

18. Van Cutsem E, Moiseyenko VM, Tjulandin S, Majlis A, Constenla $\mathrm{M}$, Boni C, et al. Phase III study of docetaxel and cisplatin plus fluorouracil compared with cisplatin and fluorouracil as first-line therapy for advanced gastric cancer: a report of the V325 Study Group. J Clin Oncol. 2006;24:4991-7.

19. Mackenzie M, Spithoff K, Jonker D, Gastrointestinal Disease Sity Group. Systemic therapy for advanced gastric cancer: a clinical practice guideline. Curr. Oncol. 2011;18:e202-e209209.
20. Bang Y-J, Van Cutsem E, Feyereislova A, Chung HC, Shen L, Sawaki A, et al. Trastuzumab in combination with chemotherapy versus chemotherapy alone for treatment of HER2-positive advanced gastric or gastro-oesophageal junction cancer (ToGA): a phase 3, open-label, randomised controlled trial. Lancet. 2010;376:687-97.

21. Canada GO, Canada H, Branch SP, Division CHA. Canada Health Act-Health Care System-Health Canada. https://www.hc-sc. gc.ca/hcs-sss/medi-assur/cha-lcs/index-eng.php.

22. Benchimol EI, Smeeth L, Guttmann A, Harron K, Moher D, Petersen I, et al. The REporting of studies Conducted using Observational Routinely-collected health Data (RECORD) statement. PLoS Med. 2015;12:e1001885-e10019221001922.

23. Robles SC, Marrett LD, Clarke EA, Risch HA. An application of capture-recapture methods to the estimation of completeness of cancer registration. J Clin Epidemiol. 1988;41:495-501.

24. Iron K, Zagorski BM, Sykora K, Manuel DG. Living and dying in Ontario: an opportunity for improved health information. ICES Investigative Report. Toronto: Institute for Clinical Evaluative Sciences; 2008.

25. Booth CM, Nanji S, Wei X, Peng Y, Biagi JJ, Hanna TP, et al. Use and effectiveness of adjuvant chemotherapy for stage III colon cancer: a population-based study. J Natl Compr Canc Netw. 2016;14:47-56.

26. Nam RK, Cheung P, Herschorn S, Saskin R, Su J, Klotz LH, et al. Incidence of complications other than urinary incontinence or erectile dysfunction after radical prostatectomy or radiotherapy for prostate cancer: a population-based cohort study. Lancet Oncol. 2014;15:223-31.

27. Rabeneck L, Paszat LF, Rothwell DM, He J. Temporal trends in new diagnoses of colorectal cancer with obstruction, perforation, or emergency admission in Ontario: 1993-2001. Am J Gastroenterol. 2005;100:672-6.

28. Kagedan DJ, Abraham L, Goyert N, Li Q, Paszat LF, Kiss A, et al. Beyond the dollar: Influence of sociodemographic marginalization on surgical resection, adjuvant therapy, and survival in patients with pancreatic cancer. Cancer. 2016;122:3175-82.

29. Karlj B. Measuring, "rurality" for purposes of health-care planning: an empirical measure for Ontario. Ont Med Rev. 2009;67:33-52.

30. Alter DA, Naylor CD, Austin P, Tu JV. Effects of socioeconomic status on access to invasive cardiac procedures and on mortality after acute myocardial infarction. N Engl J Med. 1999;341:1359-67.

31. Wilkins R. Use of postal codes and addresses in the analysis of health data. Health Rep. 1993;5:157-77.

32. Elixhauser A, Steiner C, Harris DR, Coffey RM. Comorbidity measures for use with administrative data. Med Care. 1998;36:8-27.

33. Gutacker N, Bloor K, Cookson R. Comparing the performance of the Charlson/Deyo and Elixhauser comorbidity measures across five European countries and three conditions. Eur J Public Health. 2015;25(Suppl 1):15-20.

34. Kaplan EL, Meier P. Nonparametric Estimation from Incomplete Observations. J Am Stat Assoc. 2012;6:128-32.

35. Gastwirth JL, Gel YR, Miao W. The impact of Levene's test of equality of variances on statistical theory and practice. Stat Sci. 2009;24:343-60.

36. Greenland S. Modeling and variable selection in epidemiologic analysis. Am J Public Health. 1989;79:340-9.

37. Robins JM, Greenland S. The role of model selection in causal inference from nonexperimental data. Am J Epidemiol. 1986;123:392-402.

38. Abrams TA, Brightly R, Mao J, Kirkner G, Meyerhardt JA, Schrag D, et al. Patterns of adjuvant chemotherapy use in a 
population-based cohort of patients with resected stage II or III colon cancer. J Clin Oncol. 2011;29:3255-62.

39. Cunningham D, Starling N, Rao S, Iveson T, Nicolson M, Coxon F, et al. Capecitabine and oxaliplatin for advanced esophagogastric cancer. N Engl J Med. 2008;358:36-46.

40. Okines AFC, Norman AR, McCloud P, Kang Y-K, Cunningham D. Meta-analysis of the REAL-2 and ML17032 trials: evaluating capecitabine-based combination chemotherapy and infused 5-fluorouracil-based combination chemotherapy for the treatment of advanced oesophago-gastric cancer. Ann Oncol. 2009;20:1529-34.

41. Wennberg JE. Unwarranted variations in healthcare delivery: implications for academic medical centres. BMJ. 2002;325:961-4.

42. Goodman DC. Unwarranted variation in pediatric medical care. Pediatr Clin N Am. 2009;56:745-55.

43. Mercuri M, Gafni A. Medical practice variations: what the literature tells us (or does not) about what are warranted and unwarranted variations. J Eval Clin Pract. 2011;17:671-7.

44. Sepucha K, Ozanne E, Mulley AG. Doing the right thing: systems support for decision quality in cancer care. Ann Behav Med. 2006;32:172-8.

45. Halm EA, Lee C, Chassin MR. Is volume related to outcome in health care? A systematic review and methodologic critique of the literature. Ann Intern Med. 2002;137:511-20.
46. Thiemann DR, Coresh J, Oetgen WJ, Powe NR. The association between hospital volume and survival after acute myocardial infarction in elderly patients. N Engl J Med. 1999;340:1640-8.

47. Adams EK, Wright GE. Hospital choice of Medicare beneficiaries in a rural market: why not the closest? J Rural Health. 1991;7:134-52.

48. Melnychuk M, Vindrola-Padros C, Aitchison M, Clarke CS, Fulop NJ, Levermore C, et al. Centralising specialist cancer surgery services in England: survey of factors that matter to patients and carers and health professionals. BMC Cancer. 2018;18:226.

49. Loberiza FR, Cannon AJ, Weisenburger DD, Vose JM, Moehr MJ, Bast MA, et al. Survival disparities in patients with lymphoma according to place of residence and treatment provider: a population-based study. J Clin Oncol. 2009;27:5376-82.

50. Berry SR, Bell CM, Ubel PA, Evans WK, Nadler E, Strevel EL, et al. Continental divide? The attitudes of US and Canadian oncologists on the costs, cost-effectiveness, and health policies associated with new cancer drugs. J Clin Oncol. 2010;28:4149-53.

Publisher's Note Springer Nature remains neutral with regard to jurisdictional claims in published maps and institutional affiliations. 